\title{
Using the 2016 Transit of Mercury to Find the Distance to the Sun
}

Jay M. Pasachoff, Bernd Gährken, and Glenn Schneider

Citation: The Physics Teacher 55, 137 (2017); doi: 10.1119/1.4976653

View online: http://dx.doi.org/10.1119/1.4976653

View Table of Contents: http://aapt.scitation.org/toc/pte/55/3

Published by the American Association of Physics Teachers

\section{Articles you may be interested in}

Statcast and the Baseball Trajectory Calculator

The Physics Teacher 55, (2017); 10.1119/1.4976652

3, 2, 1 ... Discovering Newton's Laws

The Physics Teacher 55, (2017); 10.1119/1.4976656

Is an Apple Magnetic: Magnetic Response of Everyday Materials Supporting Views About the Nature of Science

The Physics Teacher 55, (2017); 10.1119/1.4976654

More ideas for projecting a rainbow

The Physics Teacher 55, (2017); 10.1119/1.4976649

SELFIE STICK IMAGES

The Physics Teacher 55, (2017); 10.1119/1.4976651

Conceptualizing Rolling Motion Through an Extreme Case Reasoning Approach

The Physics Teacher 55, (2017); 10.1119/1.4976657

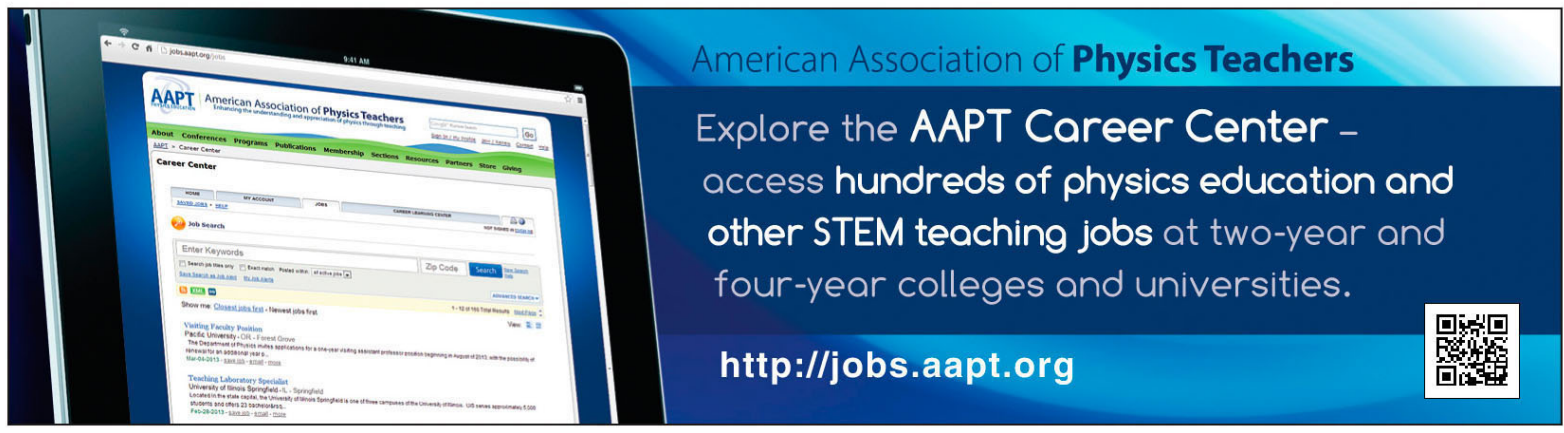




\section{Using the 2016 Transit of Mercury to Find the Distance to the Sun}

Jay M. Pasachoff, Williams College, Williamstown, MA, and Caltech, Pasadena, CA

Bernd Gährken, Bavarian Public Observatory, Munich, Germany

Glenn Schneider, Steward Observatory, The University of Arizona, Tucson, AZ

$\mathrm{T}$ he May 9, 2016, transit of Mercury was observed simultaneously from the Big Bear Solar Observatory in California and from a site in Germany. From the measured displacement between the views from the two sites of Mercury's disk silhouetted against the solar granulation, we were able to calculate the distance to the Sun in linear units (kilometers), not merely the proportionality given by Kepler's third law.

When, in 1618, Johannes Kepler in his Harmony of the World established the relation between the distances and orbital periods of the known planets as they orbit the Sun, our basic knowledge of the clockwork of the solar system was set. ${ }^{1,2}$ But all those distances were relative, with the square of the orbital periods being proportional to the cube of the distances (technically, the lengths of the semimajor axes of the orbital ellipses that Kepler had advanced in his first law, from 1609). ${ }^{3}$ The absolute calibration of the distance scale of the solar system (e.g., in physical units such as kilometers), however, remained unknown.

Kepler's Rudolphine Tables of 1627 predicted transits of Mercury and Venus across the face of the Sun in 1631, after Kepler had died. When Mercury's transit was seen, that validated not only Kepler's laws but even the Copernican heliocentric theory of planetary motion. ${ }^{4}$ The 1631 transit of Venus was not seen in Europe. But a young scientist, Jeremiah Horrocks, restudied Kepler's tables and realized that there would be a transit of Venus in 1639. Only he and one correspondent of his then saw it. ${ }^{5}$

Transits of Venus are rare: they occur in pairs separated by eight years, with then gaps of 105.5 or 121.5 years. So there were transits in 1761 (famously observed by Captain Cook from Tahiti) and 1769 and 1874 and 1882. No transits of Venus were visible from Earth in the 20th century; we have recently had a pair in 2004 and 2012. ${ }^{6,7}$ Transits of Mercury appear more often; the first was seen in 1631 by Gallendi and provided early confirmation of the accuracy of Kepler's tables. In the 21st century, there have been transits of Mercury in $2003,2006,2016$, and after the next, on Nov. 11, 2019, there will be 10 more. $^{8}$

In 1715, Edmond Halley figured out a method of finding the distance to the Sun by having observers time a transit of Venus from distances far apart from each other-in his recommendation, as far north and as far south as possible. (The predictions were not sufficiently accurate for transits of Mercury to attempt the same.) But his method required timing the entry of Venus's silhouette onto the Sun and its exit, so-called second and third contacts, to about one second of time.
It turned out that the "black-drop effect," a non-clean separation of Venus's silhouette from the solar edge, reduced the discernible time resolution to about one minute, preventing an accurate determination of the solar distance. Two of us (JMP and GS) used spacecraft observations of a 1999 transit of Mercury to finally explain the true cause of the black-drop effect, which is a composite effect related to the finite resolution of the telescope and the extreme drop-off in brightness at the edge of the Sun, which, after all, is gaseous and so has no sharp edge. ${ }^{9,10}$

In this article, we describe our use of simultaneous observations of the 2016 transit of Mercury made from two widely separated locations on Earth to determine the distance to the Sun in a way different from that suggested in 1715 by Halley. Using an internet link, teachers and students can make a similar derivation at the 2019 transit of Mercury also based on parallax and requiring only a set of measurements at one agreed-upon instant of time. We did not compare timing of the contacts (Halley's method), which groups did for the 2012 transit of Venus. ${ }^{11}$

\section{The current observations}

As with transits of Venus, transits of Mercury can also be used to determine the astronomical unit (au), which is a measure of the average distance of Earth from the Sun. Historically, however, such measurements have not been made with transits of Mercury since that planet is much smaller and farther away, giving a much lower expected accuracy. Measuring the astronomical unit from the transit of Mercury was one of the planned experiments for the May 9, 2016, transit.

One of us (BG) organized a worldwide volunteer effort to try to compare measurements of the May 9, 2016, transit of Mercury from pairs of widely separated sites on Earth. Up to

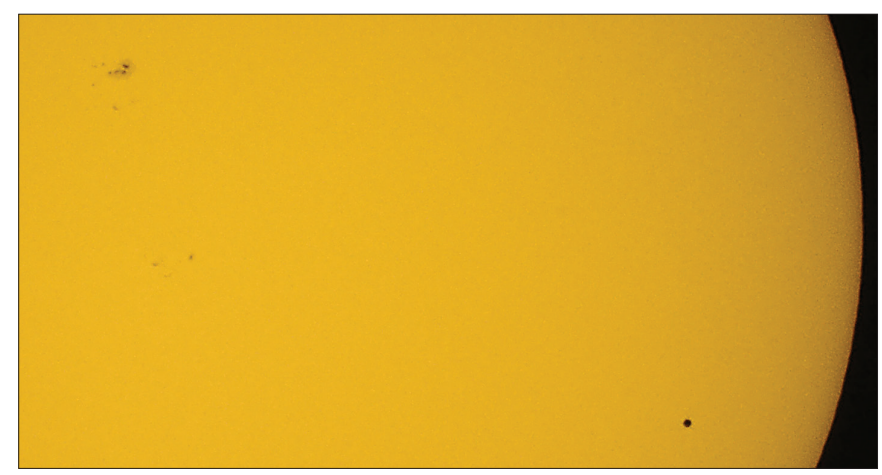

Fig. 1. A reprocessed image of the transit of Mercury of May 9, 2016, taken with a 9-cm Questar telescope and Questar filter. Note that round Mercury (lower right) is smaller even than a small-size sunspot group. 

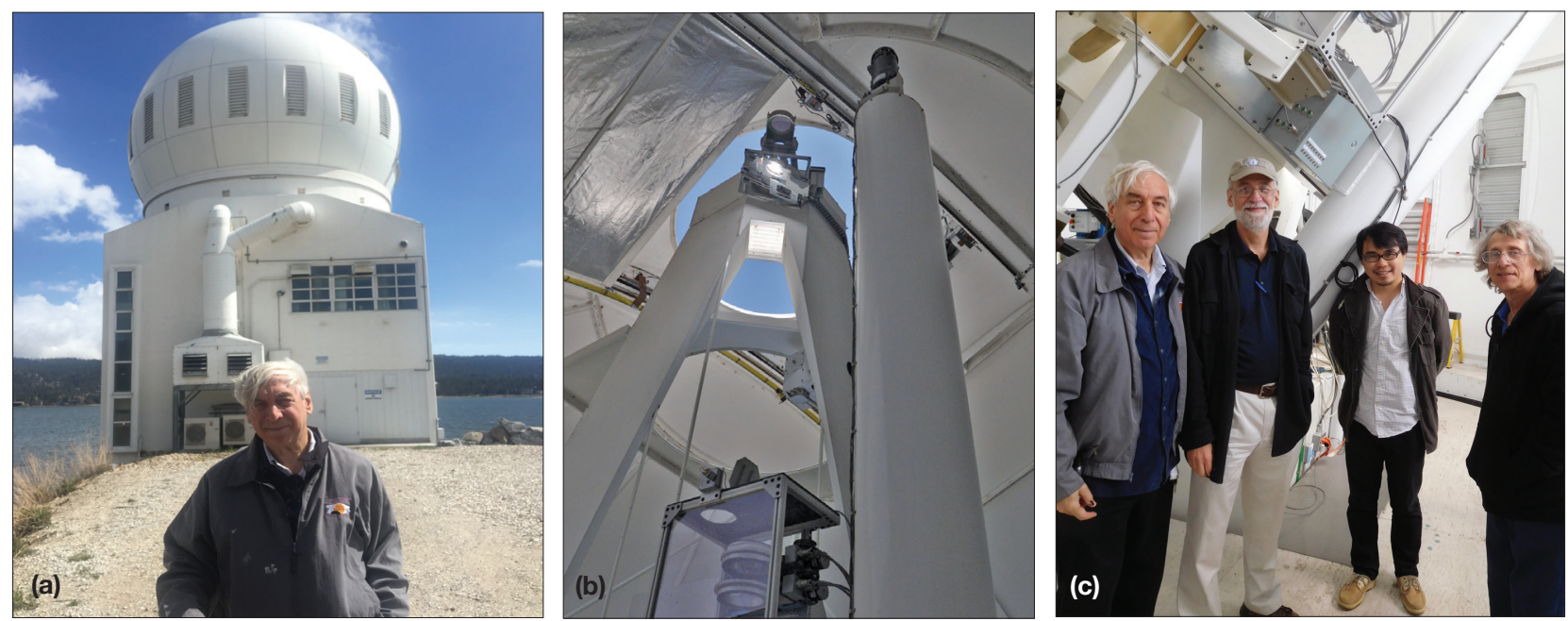

Fig. 2. The Big Bear Solar Observatory with its six-year-old New Solar Telescope, a 1.6-m off-axis reflector, is on a small artificial island in Big Bear Lake, California. (a) Author JMP is shown in front of the telescope's dome. (b) The Sun's concentrated beam is so hot that most of it is absorbed or reflected before part of it is imaged. (c) Coauthors JMP (left) and GS (right) with BBSO/NJIT faculty Dale Gary and Bin Chen in the dome.

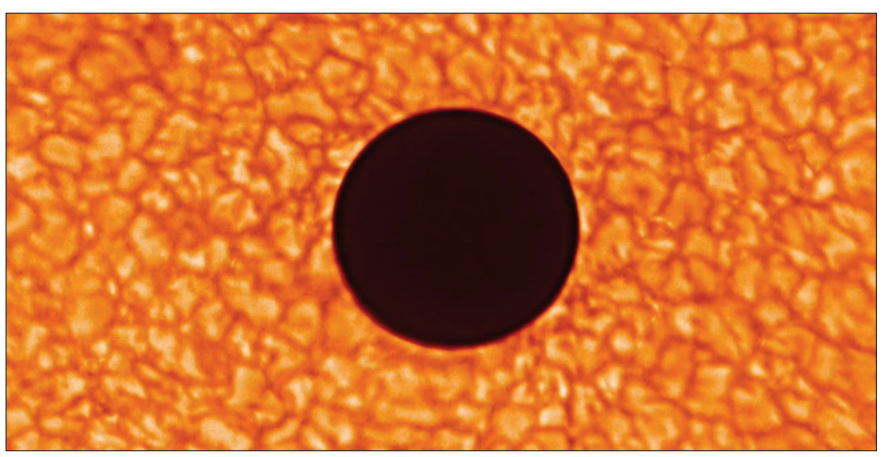

Fig. 3. Sample image from the Big Bear Solar Observatory's transit of Mercury observations, using adaptive optics. We see the Texas-sized solar granulation on the solar photosphere in the background of Mercury's opaque disk. Photo credit: Jay Pasachoff; Glenn Schneider; and Dale Gary, Vasyl Vurchyshyn, and Bin Chen of the Big Bear Solar Observatory, New Jersey Institute of Technology.

1700 UTC (Coordinated Universal Time), BG photographed the transit from Germany through an $\mathrm{H}$-alpha filter. The hope that simultaneous images could be found from an extreme southern site, such as South Africa, was not, however, fulfilled. Amateur astronomers in the Republic of South Africa responded with friendly answers and also a few pictures, but the resolution both spatially and temporally was not sufficient for the measurement.

However, two of us (JMP and GS) were using not only small telescopes (see Fig. 1 for a sample image) but also, and especially, a huge professional solar telescope: the 1.6-m off-axis reflecting New Solar Telescope of the Big Bear Solar Observatory (BBSO) of the New Jersey Institute of Technology (Latitude: $34^{\circ} 15.505^{\prime} \mathrm{N}$, Longitude: $116^{\circ} 55.278^{\prime} \mathrm{W}$ ). It is on a small artificial island off the shore of Big Bear Lake, California, with the center of the telescope's mirror at an altitude of $2067 \mathrm{~m}(6783 \mathrm{ft})$ in the mountains about two hours east of Pasadena and Los Angeles. (It is shown in Fig. 2.) Its mid-water location was chosen in the 1960s by solar-physicist
Harold Zirin of Caltech to provide steady air even at midday for solar observing.

Finally, we were able to use observations in a limitedwavelength band of visible light with the big telescope in California to compare with near-simultaneous observations taken in Germany. From 17:00 UTC onward, the filter on the German telescope was changed to a broad visual filter from an $\mathrm{H}$-alpha filter. ${ }^{12}$ The goal was to map the photospheric granulation as Mercury passed over it. The image used is the result of a video sequence made through a broadband red filter from 16:29:30 to $16: 29: 40$, with a mean of $16: 29: 35$; it is made from the 88 images with best seeing out of the sequence of 280 images. $^{13}$

Though first contact, when Mercury's silhouette first touched the solar disk, was not visible from California, from 13:00 UTC onward, when the Sun rose in California, the planet was viewable, and it rose high enough to be viewed with the BBSO telescope at about 15:00 UTC. Mercury was also seen in transit from Germany, and we hoped that comparison of simultaneous images would allow the parallax shift of simultaneous pairs of Mercury images to be measured. (Mercury had entered the solar disk from 11:12:19 to 11:15:31 UTC geocentric, passed mid-transit at 14:57:26 geocentric, and departed the solar disk from 18:39:14 to 18:42:26 UTC geocentric. ${ }^{14}$ )

Indeed, we are able to use a series of CCD observations (made also into a video) that was taken at the Big Bear Solar Observatory (BBSO) (Fig. 3). ${ }^{15}$ Two of us arranged the observations in collaboration with BBSO personnel Dale Gary, Bin Chen, Claude Plymate, Vasy Vurchyshyn, and John Varsik. The continuum observations were taken in the TiO band (705.7 nm, 10- $\AA$ bandpass).

For the measurement, we used both BBSO and German images from 16:29:35 UTC. Of course, the limiting spatial resolution from Germany was much worse, since those images were taken with only a small amateur telescope com- 


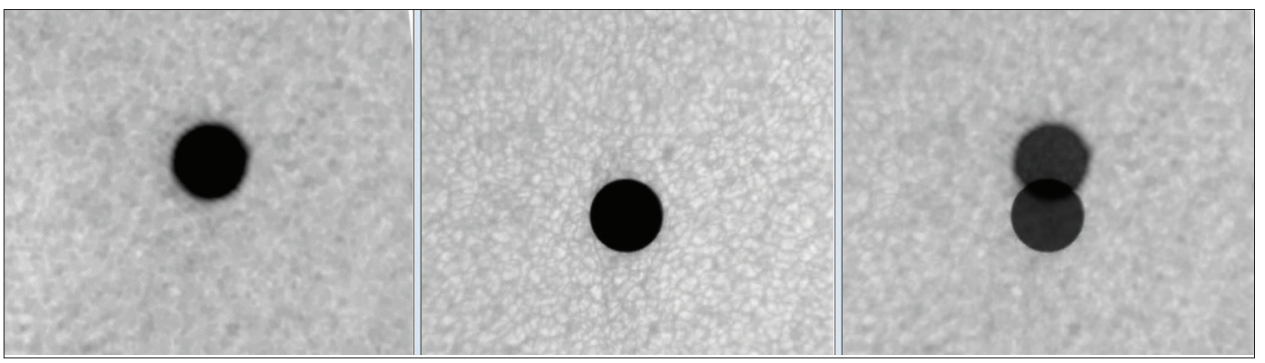

Fig. 4. Images at relatively low resolution (left) could easily be aligned with contemporaneous high-resolution images from the Big Bear Solar Observatory 1.6-m telescope observations (center), by correlating patterns in the background solar granulation, to reveal the offset in the positions of Mercury from the two different positions on Earth.

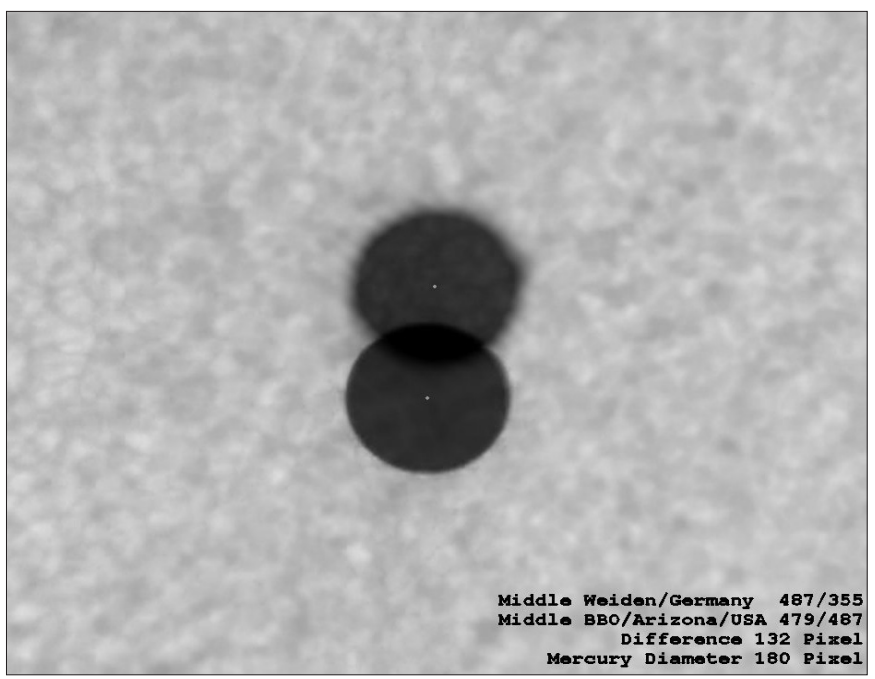

Fig 5. The difference in resolution is visible between the small telescope in Weiden, Germany (top), and the large telescope of the Big Bear Solar Observatory (bottom) for Mercury's silhouette, but alignment on the granulation was easy, since the images were taken simultaneously so the displacement was small. The difference is 132 pixels, compared with Mercury's diameter of 180 pixels. (Disk centers were measured, in $x-y$ by pixels, at $4877 / 355$ for Weiden and 479/487 for Big Bear.)

pared with the Big Bear Solar Observatory's large professional telescope, additionally improving image fidelity at BBSO with adaptive optics. Nevertheless, the displacement of Mercury's image was so small against the background granulation that the two images are convincingly aligned and overlain by inspection (as shown in Figs. 4 and 5).

From Fig. 5, we measured the relative displacement (parallactic shift) of the location of Mercury with respect to the solar photosphere as seen from our two different locations on Earth. We compared the offset of the two disks with the to the direction to the Sun. angular size of Mercury as measured from the two images resampled to a common pixel scale.

The distance between the sites in Weiden, in Germany, and Big Bear Solar Observatory in California, measured along the surface of Earth, was determined to be $9416 \mathrm{~km}$, or a shorter $8587.7 \mathrm{~km}$ in space pointto-point [see Fig. 6(a)]. This value would correspond to the direction of the Sun if it were at the same angular distance above the horizon at both

locations. However, the German measurements were with the Sun $19.35^{\circ}$ above the horizon while the BBSO measurements were made with the Sun $43.7^{\circ}$ above the horizon. The difference of $24.35^{\circ}$ shortens the line of sight from the perspective of the Sun to $\cos \left(24.35^{\circ}\right)^{\star} 8587.7=7824 \mathrm{~km}$ (Fig. 6).

We can then use the parallactic shift we measured as 8.86 arcseconds with the terrestrial baseline difference of 7824 to compute the solar distance.

\section{Parallax and the calculation of distance}

Astronomers often define distances in terms of an angle of parallax. For example, the parallax of the Sun corresponds to the half-angle subtended by Earth's disk from the point of view of an observer at the center of the Sun. (For stellar distances, stellar parallaxes are the half-angle subtended by the radius of the Earth's orbit as seen from the star, and are all less than one second of arc, preventing them from being measured until the 19th century.) The difference between the parallax measured from the center of the Sun and the parallactic shift measured with respect to the solar photosphere is small (for Mercury at the center of the solar disk, 700,000 $\mathrm{km}$ for the solar radius divided by $\sim 150,000,000 \mathrm{~km}$ for the solar distance $=\sim 7 / 15 \%=\sim 0.5 \%$ ).

Given our measured solar parallax at the UTC of the transit, specifically at the time of the images we used, as 8.86 arcseconds, using the diameter of $12,757 \mathrm{~km}$ for Earth, we can
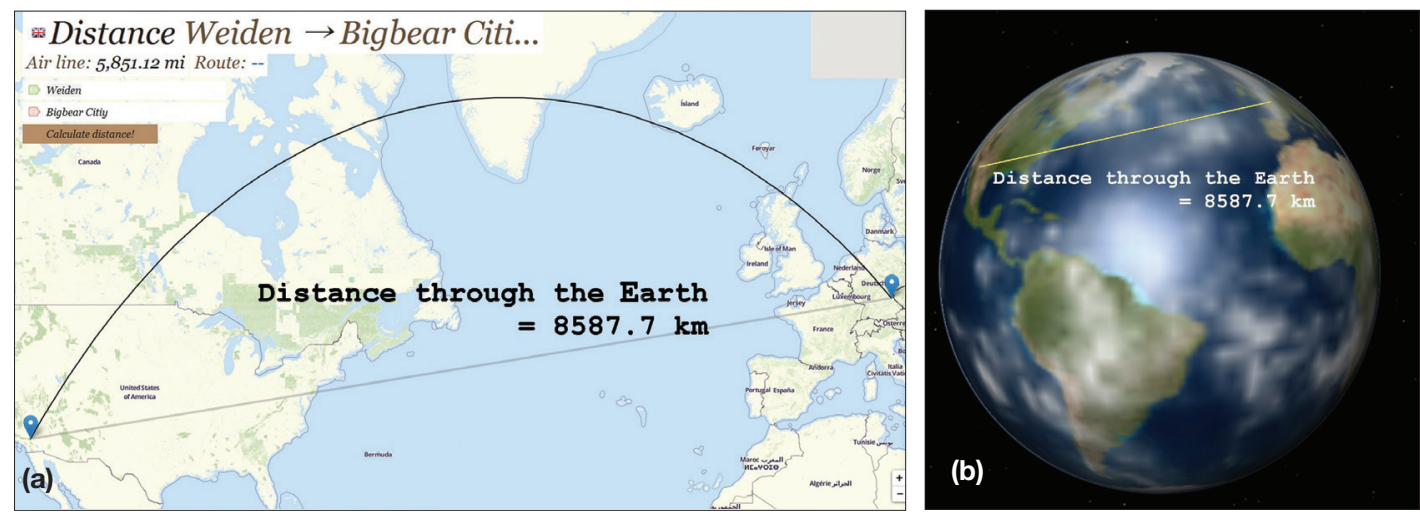

Fig. 6. (a) To calculate the astronomical unit using the concept of parallax, we use a long, skinny triangle having a baseline of the distance between two locations on Earth and perpendicular to the direction to the Sun. (b) The baseline for the calculation is the distance between two locations on Earth and perpendicular 


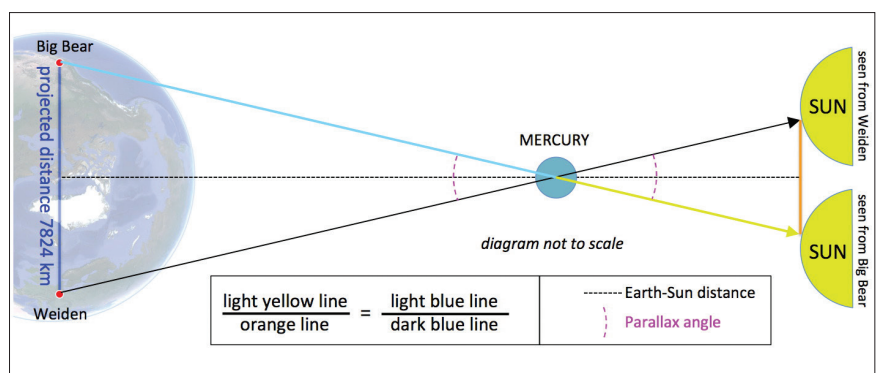

Fig. 7. Determination of the Earth-Sun distance at the time of the transit, using the concept of parallax with a long, skinny triangle having a baseline of the distance between two locations on Earth, a key point being that the parallax angle is, of course, equal to the left and to the right of the vertex at Mercury. (The diagram is not to scale; at the right, the actual displacement is a small fraction of the solar diameter, but it is greatly exaggerated here for clarity.)

now calculate the distance of Earth from the Sun at that date and time directly from the solar parallax (Fig. 7).

Though we calculated the angle for the projected distance between our two terrestrial sites, as shown in the proportions in the equation in the figure, the solar parallax is defined as the angle subtended by Earth's radius from the Sun, which corresponds to the views from opposite sides of Earth divided by two to give the terrestrial radius. So the Earth-Sun distance $=$ diameter of Earth $/ \tan [$ (measured solar parallax in arcseconds/ 3600) *2]. The result is a value of 148.5 million $\mathrm{km}$ to the photosphere, or $\sim 148.9$ million $\mathrm{km}$ to the solar center, for which the solar parallax is officially defined.

The International Astronomical Union defines the astronomical unit, which is approximately the average distance from Earth to the Sun, and in 2015 they changed its notation from $\mathrm{AU}$ to au. ${ }^{16}$ To determine the astronomical unit in linear units like kilometers, we first calculate the distance from Mercury to the Sun in au, using the orbital period and Kepler's third law. The orbit of Mercury, however, is highly (with respect to that of other planets) elliptical. Therefore, the actual distance is variable. For simplicity we used a value from the PC/Mac/Linux planetarium program Cartes du Ciel (http://www.ap-i.net/skychart/en/; note hyphen in "ap-i"; it is not to be confused with the international effort Carte du Ciel begun in the 19th century). ${ }^{17}$ Accordingly, the distance from the center of the Sun to Mercury on the day and time of the simultaneous transit observations in Weiden and Big Bear was $0.4529 \mathrm{au}^{18}$

Because of Earth's elliptical orbit, the distance to the Sun on the day of the transit was larger than the astronomical unit of 149.6 million $\mathrm{km}$; it was 1.0097 au or $151,050,000 \mathrm{~km}$. Our value is therefore $(151.05-148.9)$ million $\mathrm{km}=2.15$ million $\mathrm{km}$, or about $2.15 / 151=<2 \%$ from the known value.

We attribute the uncertainty mainly to the measurement error in the observational determination of the displacement of Mercury's silhouette between the two images.

A similar comparison was made at the 2004 transit of Venus between Munich and Hong Kong: http://www.astrode.de/ venustr2.htm. See also the parallax measurements organized by Udo Backhaus of Universität Duisberg-Essen, Germany, in which we collaborated: http://www.venus2012.de/transit-ofmercury2016/results.php.

\section{Exoplanet analogues}

This paper uses Kepler's third law (1618) to relate the distance to Mercury and the distance to the Sun, so it is a 21 st-century tribute to Kepler's work. The method of detecting planetary transits, first calculated from Johannes Kepler's Rudolphine Tables (1627) and using Kepler's laws for interpretation, led to NASA's naming a planet-hunting satellite Kepler, now in the $K 2$ version of its extended mission. The Kepler/K2 missions have discovered thousands of exoplanets, planets around other stars, by the transit method. ${ }^{19,20}$

\section{Summary}

We have used simultaneous measurements made at opposite sides of Earth during the 2016 transit of Mercury across the face of the Sun to measure the apparent displacement of the planet's silhouette against the visible solar granulation. We used that angular displacement to calculate the distance at that moment between Earth and the Sun (actually, the solar photosphere).

\section{Acknowledgments}

We acknowledge the collaboration at the Big Bear Solar Observatory of its faculty and staff, including Dale Gary, Bin Chen, Claude Plymate, Vasyl Vurchyshyn, and John Varsik. We were pleased to have Robert Lucas (Sydney) and Evan Zucker (San Diego) join us for transit observations. We thank Udo Backhaus of Universität Duisberg-Essen, Germany, for his comments.

\section{References}

1. N. Pasachoff and J. M. Pasachoff, "Kepler," in The Scientists: An Epic of Discovery, edited by Andrew Robinson (Thames and Hudson, London, 2012), pp. 26-31.

2. J. Voelkel, Johannes Kepler and the New Astronomy (Oxford University Press, New York, 2000), pp. 131-132.

3. J. M. Pasachoff and A. Filippenko, The Cosmos: Astronomy in the New Millennium, 4th ed. (Cambridge University Press, New York, 2014).

4. O. Gingerich, "Transits in the seventeenth century and the credentialing of Keplerian astronomy," J. Hist. Astr. 44 (3), 303-312 (2013).

5. J. M. Pasachoff, "Catch a pass! (of Venus with the Sun)," Odyssey, 40-42 (May/June 2011).

6. J. Westfall and W. Sheehan, Celestial Shadows: Eclipses, Transits, and Occultations (Springer, New York, 2015).

7. J. M. Pasachoff, "Transit of Venus: Last chance from Earth until 2117," Phys. World 25 (5), 36-41 (May 2012).

8. http://eclipsewise.com/oh/tm2016.html .

9. G. Schneider, J. M. Pasachoff, and L. Golub, “TRACE Observations of the 15 November 1999 transit of Mercury and the black drop effect: Considerations for the 2004 Transit of Venus,' Icarus 168, 249-256 (2004).

10. J. M. Pasachoff, G. Schneider, and L. Golub, "The black-drop effect explained," in Transits of Venus: New Views of the Solar System and Galaxy, IAU Colloquium No. 196, edited by D. W. Kurtz and G. E. Bromage (Cambridge University Press, Preston, Lankashire, U.K., 2005), pp. 242-253. 
11. J. K. Faherty, D. R. Rodriguez, and S. T. Miller, “Te Hetu'u Global Network: Measuring the distance to the Sun using the June 5th/6th transit of Venus," Astr. Educ. Rev. 11 (1), (Dec. 2012); http://portico.org/stable?au=pgg3ztfbrn3. See also https://arxiv.org/abs/1210.0873. Prof. Udo Backhaus's project page at http://www.venus2012.de/venusprojects/contacttimes/ contacttimes.php discusses the method using contact times instead of the parallax that is the subject of the current paper.

12. An $800 \mathrm{~mm} \mathrm{f} / 15$ refractor two-element acromat was used with a Herschel wedge to diminish the solar brightness. The Weiden site was at latitude $49.6744^{\circ}$ and longitude $12.1489^{\circ}$.

13. The camera was an Omegon Proteus 120 MSI (Aptina MT9M034 Chip).

14. F. Espenak, http://eclipsewise.com/oh/tm2016.html .

15. http://www.bbso.njit.edu/scinews/AIA_NST_Mercury_ Transit_with_title.mp4.

16. According to its definition adopted by the XXVIIIth General Assembly of the IAU (IAU 2012 Resolution B2), the astronomical unit is a conventional unit of length equal to 149597 870700 m exactly.

17. JPL's Horizons ephemeris with DE431 mx gives the same value; http://ssd.jpl.nasa.gov/?horizons, with calculations at http:// ssd.jpl.nasa.gov/horizons.cgi.

18. Anyone interested in the calculation according to Kepler can look at the website http://www.astrode.de/ merkurtransit/5merkurt9be.htm, available in both German and English. For a more detailed calculation of the distance to the Sun, thanks to Prof. Udo Backhaus, see the " 2016 transit of Mercury" section of http://transitofvenus.info.

19. J. M. Pasachoff, audio tours at Smithsonian's National Air and Space Museum: "What did Johannes Kepler find out about planetary orbits?" (\#3); "How do astronomers today use Johannes Kepler's findings about planetary orbits?” (\#48), http:// airandspace.si.edu/exhibitions/explore-the-universe/audiotour/index.cfm, or s.si.edu/ETUaudio; transcript: http:// airandspace.si.edu/files/pdf/exhibitions/etu-audio-transcript. pdf (2015).

20. G. Schneider, J. M. Pasachoff, and R. C. Willson, "The effect of the transit of Venus on ACRIM's total solar irradiance measurements: Implications for transit studies of extrasolar planets," Astrophys. J. 641, 565-571 (2006).

Jay Pasachoff is an astronomer at Williams College; the observations were made and this paper was written during a sabbatical at Caltech. Bernd Gährken is a weekend astronomer who organized the worldwide effort. Glenn Schneider is an astronomer at The University of Arizona. Pasachoff and Schneider have studied four transits of Mercury and two of Venus as seen from Earth, and explained the cause of the black-drop effect. jay.m.pasachoff@ williams.edu; bgaehrken@web.de; gschneider@as.arizona.edu; http://www. sternwarte-muenchen.de/portrait_e. html; see http://www.astrode.de/ merkurtransit/5merkurt9be.htm
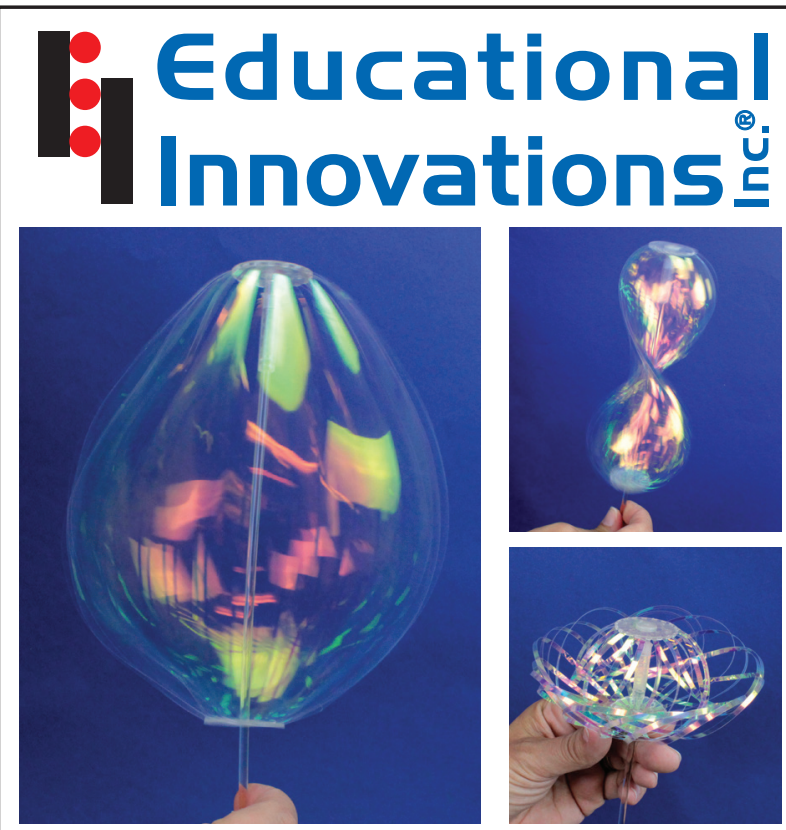

\section{Centripetal Spinner}

The prettiest demonstration of centripetal force and inertia we've ever seen! This perky, iridescent device reflects a dazzling rainbow as it spins. Twirl the stick and the thin ribbons spread into a bubble shape. The faster you spin, the wider the bubble becomes! It can be gently twisted by hand to make a delicate "flower" that neatly tucks itself into a tight ball. Endlessly fascinating! Lessons and video on our website!

PHY-250 \$3.95

Shipping charges will be added to final order. See our website for shipping rates.

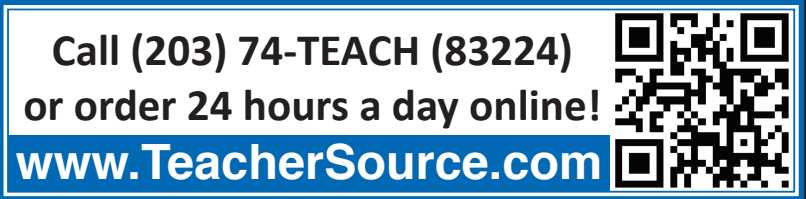

\section{Seeing Light in a Whole New Way}

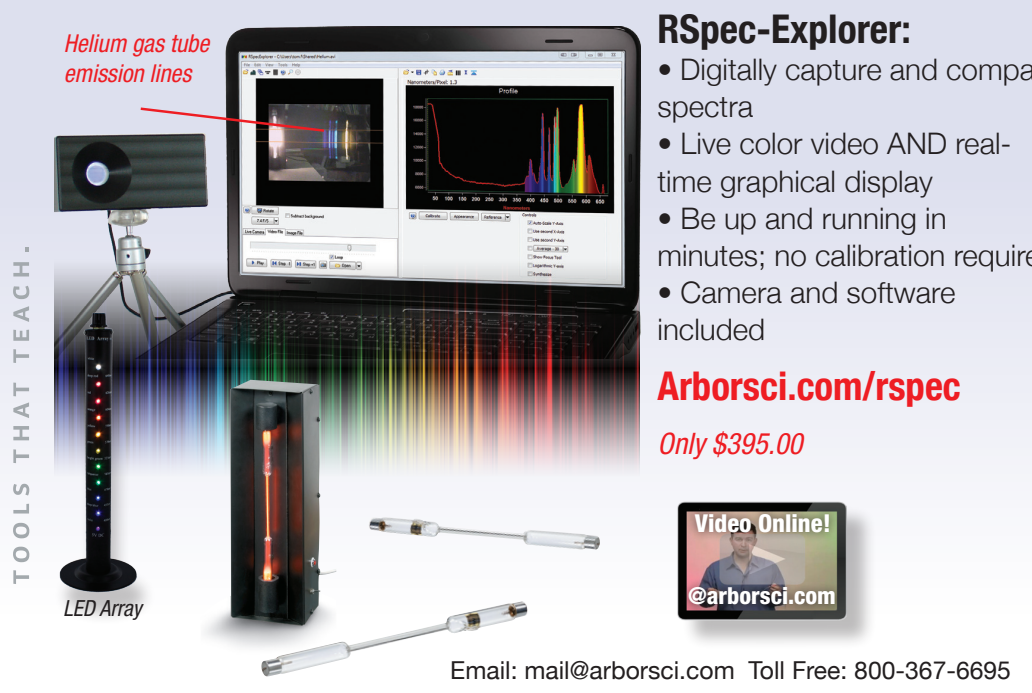

